\title{
Breaking Wave Spectrum and Set-Down Set-Up in Shallow Water
}

W.H. Yang

Department of Harbor and River Engineering National Taiwan Ocean University Keelung, Taiwan, R.O.C.

Follow this and additional works at: https://jmstt.ntou.edu.tw/journal

Part of the Civil and Environmental Engineering Commons

\section{Recommended Citation}

Yang, W.H. (1993) "Breaking Wave Spectrum and Set-Down Set-Up in Shallow Water," Journal of Marine Science and Technology. Vol. 1: Iss. 1, Article 11.

DOI: $10.51400 / 2709-6998.2481$

Available at: https://jmstt.ntou.edu.tw/journal/vol1/iss1/11

This Research Article is brought to you for free and open access by Journal of Marine Science and Technology. It has been accepted for inclusion in Journal of Marine Science and Technology by an authorized editor of Journal of Marine Science and Technology. 


\title{
BREAKING WAVE SPECTRUM AND SET-DOWN SET-UP IN SHALLOW WATER
}

\author{
W.H.Yang \\ Department of Harbor and River Engineering \\ National Taiwan Ocean University \\ Keelung, Taiwan, R.O.C.
}

Key Words: breaking wave spectrum, set-up set-down, radiation stress.

\begin{abstract}
To study the mean water surface in the shallow water, it is necessary to compute the wave set-up and set-down of random waves on the surf zone. Therefore, the breaking wave spectra and radiation stress must be calculated from a wide-band breaking wave model.

In this study, we extend the breaking wave spectrum model (Yang, 1991) by using the Wallops spectrum in the deep water. The Wallops spectrum is based on wave dynamics theoretical analysis and no empirical coefficients to be determined by the users. It also contents correct energy (Huang et al., 1981). The breaking wave spectra, radiation stress, and set-down set-up are presented graphically with distance from shoreline. It is shown that the results agree with the model (Yang, 1991) which used Pierson spectrum in the deep water.
\end{abstract}

\section{INTRODUCTION}

In order to study the set-down and set-up of irregular waves on sloping beaches, it must compute the radiation stress and breaking spectrum of waves in the shallow water. The shallow water breaking wave spectrum was studied by Yang (1991) which used Pierson spectrum in the deep water. In this study, we used Wallops spectrum which is involved wave theory analysis and correct energy content (Huang et al., 1981). However, the Wallops spectrum does not consider wave breaking. The modification of breaking Wallops spectrum in deep water was studied by Tung et al. (1987). Although, the importance of the breaking waves and radiation stress must be consider in shallow water. There are numbers of publications on the subject of breaking waves in shallow water such as Battjes and Janssen (1978). However these methods do not obtain the breaking wave spectrum directly.

By improving the breaking wave criterion used in Battjes (1974), a wide-band model of breaking waves approach to the shoreline is established. Based on the model and assuming the original ideal (fictitious) non-breaking waves are zero-mean, stationary, and Gaussian, the spectrum of breaking waves is derived and the corresponding radiation stress is computed. The variances of the spectrum and the standard deviations of wave elevation and radiation stress with distance from shoreline are computed and presented graphically. It is shown that the results are in good agreement with those data by Yang (1991) and Yang and Tung (1993).

\section{PROBLEM STATEMENT}

Breaking criterion for sinusoidal and finite bandwidth waves is (Yang, 1991)

$$
\zeta_{b}=0.44 d \frac{\tanh \left(k_{0} d\right)}{k_{0} d}
$$

where $d$ is water depth, $k_{0}$ is the component wavenumber in deep water for waves of finite-bandwidth. For simplicity, it is assumed that $k_{\mathrm{o}}$ in Eq. (1) is the mean wave-number in deep water denoted $\bar{k}_{0}$ and is 
related to the mean wave-frequency $\bar{a}_{0}$ in deep water by the dispersive relation $\bar{k}_{0}=\frac{\bar{\omega}_{0}{ }^{2}}{g}, g$ being gravita tional acceleration. Therefore, we assume the ideal (fictitious) wave amplitude breaks and reaches $\zeta_{b}$ if water surface elevation $\zeta(t)$ exceeds $\zeta_{b}$. The mean water surface elevation $\bar{\zeta}(t)$ can be considered as

$$
\begin{aligned}
\bar{\zeta}= & \zeta \times H\left(\zeta_{b}-\zeta\right) \times H(\zeta)+\zeta_{b} \times H\left(\zeta-\zeta_{b}\right)+\zeta \\
& \times H(d+\zeta) \times H(-\zeta)-d \times H(-d-\zeta)
\end{aligned}
$$

where $H(\cdot)$ is the Heaviside step function and $\zeta(t)$ is elevation of the ideal waves as in Fig. 1. The wave elevation in Eq. (2) is a nonlinear function of the random process $\zeta(t)$. Assuming that $\zeta(t)$ is zero-mean, stationary, and Gaussian (Phillips 1977). The expected mean water surface considering breaking $\mathrm{E}[\bar{\zeta}]$ is

$$
\begin{aligned}
E[\bar{\zeta}]= & E\left[\zeta \times H(\zeta) \times H\left(\zeta_{b}-\zeta\right)\right]+\zeta_{b} \\
& \times E\left[H\left(\zeta-\zeta_{b}\right)\right]+E[\zeta \times H(-\zeta) \times H(d+\zeta)] \\
& -d \times E[H(-d-\zeta)]
\end{aligned}
$$

Here, $\mathrm{E}[\cdot]$ is the expected value of the quantity in the bracket. Eq. (3) can be transformed (Yang, 1991) into

$$
E[\bar{\zeta}]=\sigma[Z(\bar{\eta})-Z(\bar{\eta})-\bar{\eta} Q(\bar{\eta})+\bar{\eta} Q(\bar{\eta})]
$$

where $\sigma$ is the standard deviation of $\zeta(t), \bar{\eta} \equiv \frac{\zeta_{b}}{\sigma}$ and $\tilde{\eta} \equiv \frac{d}{\sigma}$ respectively. The function $Z(\lambda)$ and $Q(\lambda)$ are defined as

$$
Z(\lambda)=\frac{1}{(2 \pi)^{1 / 2}} \exp \left[-\frac{\lambda^{2}}{2}\right]
$$

and

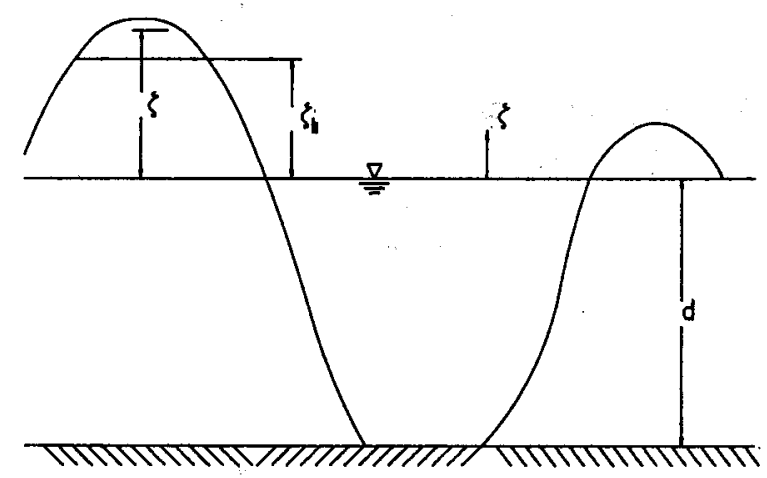

$$
Q(\lambda)=\int_{\lambda}^{\infty} Z(s) d s
$$

The standard deviation of ideal wave elevation at specified water depth is given by

$$
\sigma^{2}=\int_{0}^{\infty} S(\omega) d \omega
$$

where $S(\omega)$ is ideal wave spectrum in the specified water depth. In this study, we chose the Wallops spectrum for deep water spectrum. Because it is based on fluid dynamic analysis with no empirical coefficients and correct energy content (Huang et al. 1981). Therefore, the deep water spectrum is

$$
S_{0}(\omega)=\frac{\beta g^{2}}{\omega^{m} \omega^{5-m}} \exp \left[-\frac{m}{4}\left(\frac{\omega_{0}}{\omega}\right)^{4}\right]
$$

where $\beta$ and $m$ are function of the significant slope $\S$ of wave field and $\omega_{0}$ is the frequency of the waves at spectral peak. The significant slope $\S$ is defined as

$$
\S=\frac{M_{0}^{1 / 2}}{\lambda_{0}}
$$

where $M_{\mathrm{o}}$ is the zero-moment of the Wallops spectrum and $\lambda_{0}=\frac{2 \pi}{k_{0}}$ is the wave length corresponding to the waves at the spectrum peak. The two parameters, $m$ and $\beta$, in the Wallops spectrum then define as

$$
m=\frac{\log (\sqrt{2} \pi \S)^{2}}{\log 2}
$$

and

$$
\beta=\frac{m^{(m-1) / 4}}{4^{(m-5) / 4}} \frac{(2 \pi \S)^{2}}{\Gamma\left[\frac{(m-1)}{4}\right]}
$$

where $\Gamma[\cdot]$ is the Gamma function.

In this study, we consider the beach is infinite long with parallel depth contours monotonically decreasing water depth to the shore ward direction. The incoming waves are long-crested and progressing parallel to the water depth contour. The shoaling spectrum of finite water depth $S(\omega)$ can be obtained from Battjes (1974)

$$
S(\omega)=S_{0}(\omega) \frac{C_{g 0}}{C_{g}}
$$

Fig. 1. Schematic Diagram of Breaking Wave. 
where $C_{g}$ and $C_{g 0}$ are group velocities in deep water and finite water depth respectively. Using the approximation of component wave number in deep water $k_{\mathrm{o}}$ and in the finite water depth $k$ (Tayfun, 1979),

$$
k_{0} \cong k \tanh ^{1 / 2}\left(k_{0} d\right)
$$

the shoaling coefficient of wave spectrum can be represented

$$
\frac{C_{g 0}}{C_{g}}=\frac{\tanh ^{1 / 2}\left(k_{0} d\right)}{\tanh \left(k_{0} d\right)+k_{0} d\left[1-\tanh \left(k_{0} d\right)\right]}
$$

The standard deviation of ideal (fictitious) waves $\sigma$ can be calculated from Eq. (7), (12) and (14).

Similar to the mean value of breaking wave water surface, the mean-square value of $\bar{\zeta}$ can be given as

$$
\begin{aligned}
E\left[\bar{\zeta}^{2}\right]= & \sigma^{2}\left[1+\left(\bar{\eta}^{2}-1\right) \times Q(\bar{\eta})+\left(\bar{\eta}^{2}-1\right)\right. \\
& \times Q(\bar{\eta})-\bar{\eta} \times Z(\bar{\eta})-\bar{\eta} \times Z(\overline{\bar{\eta}})]
\end{aligned}
$$

The standard deviation of $\bar{\zeta}$ can be calculated as

$$
\sigma_{\zeta}^{2}=E\left[\bar{\zeta}^{2}\right]-E^{2}[\bar{\zeta}]
$$

In order to obtain the breaking wave spectrum the auto-correlation function $R_{\bar{\zeta} \bar{\zeta}}$ and covariance function $K_{\bar{\zeta}}$ must be calculated first. Because the $\bar{\zeta}(t)$ is stationary and letting subscripts 1 and 2 refer to time instants $t+\tau$ and $t$ respectively, so

$$
\begin{aligned}
R_{\bar{\zeta} \bar{\zeta}}(\tau)= & E\left[\bar{\zeta}_{1} \bar{\zeta}_{2}\right]=E\left[\left(\zeta_{1} \times H\left(\zeta_{b}-\zeta_{1}\right) \times H\left(\zeta_{1}\right)\right.\right. \\
& +\zeta_{b} \times H\left(\zeta_{1}-\zeta_{b}\right)+\zeta_{1} \times H\left(d+\zeta_{1}\right) \\
& \left.\times H\left(-\zeta_{1}\right)-d \times H\left(-d-\zeta_{1}\right)\right) \times\left(\zeta_{2}\right. \\
& \times H\left(\zeta_{b}-\zeta_{2}\right) \times H\left(\zeta_{2}\right)+\zeta_{b} \times H\left(\zeta_{2}-\zeta_{b}\right) \\
& +\zeta_{2} \times H\left(d+\zeta_{2}\right) \times H\left(-\zeta_{2}\right)-d \\
& \left.\left.\times H\left(-d-\zeta_{2}\right)\right)\right]
\end{aligned}
$$

Cross-multiplying and taking the expected values on the right hand side of Eq. (17) give rise to sixteen expectations of which, because of symmetry, only ten terms need to be evaluated. While these expected values can all be obtained in a straight forward manner it is expedient to utilize the Hermite polynomial representation of the joint probability density function of the zero-mean Gaussian random variables $\zeta_{1}$ and $\zeta_{2}$

$$
\begin{aligned}
f_{\zeta_{1} \zeta_{2}}\left(\zeta_{1} \zeta_{2}\right)= & \frac{1}{2 \pi\left(1-r^{2}\right)^{1 / 2} \sigma^{2}} \\
& E X P\left[-\frac{1}{2(1-r)^{2}}\left[\left(\frac{\zeta_{1}}{\sigma}\right)^{2}+\left(\frac{\zeta_{2}}{\sigma}\right)^{2}\right.\right. \\
& \left.\left.-2 r\left(\frac{\zeta_{1} \zeta_{2}}{\sigma^{2}}\right)\right]\right]
\end{aligned}
$$

where

$$
r(\tau)=\frac{E\left[\zeta_{1} \zeta_{2}\right]}{\sigma^{2}}=\frac{R(\tau)}{\sigma^{2}}
$$

is the correlation coefficient of $\zeta_{1}$ and $\zeta_{2}, R(t)$ being the auto correlation function of $\zeta$.

From Erdely et al. (1953), the standard joint prob-ability density function $f_{\eta_{1}} \eta_{2}\left(\eta_{1} \eta_{2}\right)$ of zeromean Gaussian random variables $\eta_{1}$ and $\eta_{2}$ may be expressed in term of Hermite polynomial in series form as

$$
\begin{aligned}
f_{\eta_{1} \eta_{2}}\left(\eta_{1} \eta_{2}\right)= & \frac{1}{2 \pi\left(1-r^{2}\right)^{1 / 2}} E X P\left[-\frac{1}{2\left(1-r^{2}\right)}\right. \\
& \left.\left(\eta_{1}^{2}+\eta_{2}^{2}-2 r \eta_{1} \eta_{2}\right]\right] \\
= & \sum_{n=1}^{\infty} r^{n} h_{n}\left(\eta_{1}\right) h_{n}\left(\eta_{2}\right) Z\left(\eta_{1}\right) Z\left(\eta_{2}\right)
\end{aligned}
$$

where

$$
h_{n}(\eta)=\frac{(-1)^{2}}{(n !)^{1 / 2}} \operatorname{EXP}\left(\frac{\eta^{2}}{2}\right) \frac{d^{n}}{d \eta^{n}} E X P\left(-\frac{\eta^{2}}{2}\right)
$$

is the Hermite polynomial. Thus, for example,

$$
\begin{gathered}
h_{0}(\eta)=1, \quad h_{1}(\eta)=\eta, \quad h_{2}(\eta)=\left(-1+\eta^{2}\right) / \sqrt{2} \\
h_{3}(\eta)=-\left(3 \eta-\eta^{3}\right) / \sqrt{6} \text { etc. }
\end{gathered}
$$

Upon substituting $\eta_{1}=\frac{\zeta_{1}}{\sigma}$ and $\eta_{2}=\frac{\zeta_{2}}{\sigma}$ and using the expansion in Eq. (21), all the expected values on the right-hand-side of Eq. (17) may be expressed in terms of the following integrals:

$$
I_{n}(\lambda)=\int_{\lambda}^{\infty} h_{n}(\eta) Z(\eta) d \eta
$$


and

$$
J_{n}(\lambda)=\int_{0}^{\lambda} \eta h_{n}(\eta) Z(\eta) d \eta
$$

These integrals may all carried out and expressed in terms of $Z(\cdot)$ and $Q(\cdot)$ and recursive formula may be derived but these are not shown here. The expected values on the right-hand-side of Eq. (17) also involve integrals of the form

$$
\int_{-\infty}^{-\lambda} h_{n}(\eta) Z(\eta) d \eta=(-1)^{n} I_{n}(\lambda)
$$

and

$$
\int_{-\lambda}^{0} \eta h_{n}(\eta) Z(\eta) d \eta=(-1)^{n+1} J_{n}(\lambda)
$$

which are therefore expressible in terms of $Z(\bullet)$ and $Q(\bullet)$.

Thus, for example, the expected value

$$
\begin{aligned}
E_{1}= & E\left[\zeta_{1} \zeta_{2} \times H\left(\zeta_{b}-\zeta_{1}\right) \times H\left(\zeta_{b}-\zeta_{2}\right) \times H\left(\zeta_{1}\right)\right. \\
& \left.\times H\left(\zeta_{2}\right)\right] \\
& =\int_{\zeta_{1}=0}^{\zeta_{b}} \int_{\zeta_{2}=0}^{\zeta_{b}} \zeta_{1} \zeta_{2} f_{\zeta_{1} \zeta_{2}}\left(\zeta_{1} \zeta_{2}\right) d \zeta_{1} d \zeta_{2} \\
& =\sigma^{2} \int_{\eta_{1}=0}^{\bar{\eta}} \int_{\eta_{2}=0}^{\bar{\eta}} \eta_{1} \eta_{2} f_{\eta_{1} \eta_{2}}\left(\eta_{1} \eta_{2}\right) d \eta_{1} d \eta_{2} \\
& =\sigma^{2} \sum_{n=0}^{\infty} r^{n}\left[\int_{\eta=0}^{\bar{\eta}} \eta h_{n}(\eta) Z(\eta) d \eta\right]^{2} \\
& =\sigma^{2} \sum_{n=0}^{\infty} r^{n} J_{n}^{2}(\bar{\eta})
\end{aligned}
$$

similarly,

$$
\begin{aligned}
E_{2} & =E\left[\zeta_{1} \times H\left(\zeta_{b}-\zeta_{1}\right) \times H\left(\zeta_{1}\right) \times H\left(\zeta_{2}-\zeta_{b}\right)\right] \\
& =\int_{\zeta_{1}=0}^{\zeta_{b}} \int_{\zeta_{2}=\zeta_{b}}^{\infty} \zeta_{1} f_{\zeta_{1} \zeta_{2}}\left(\zeta_{1} \zeta_{2}\right) d \zeta_{1} d \zeta_{1} \\
& =\sigma \int_{\eta_{1}=0}^{\bar{\eta}} \int_{\eta_{2}=\pi}^{\infty} \eta_{1} f_{\eta_{1} \eta_{2}}\left(\eta_{1} \eta_{2}\right) d \eta_{1} d \eta_{2} \\
& =\sigma \sum_{n=0}^{\infty} r^{n} J_{n}(\bar{\eta}) I_{n}(\eta)
\end{aligned}
$$

The remaining expected values on the righthand-side of Eq. (17) may be all be written down in a routine manner without effort and will not be shown. The final result is

$$
R_{\bar{\zeta} \bar{\zeta}}(\tau)=\sigma^{2} \sum_{n=0}^{\infty} r^{n}\left(\hat{I}_{n}+\hat{J}_{n}\right)^{2}
$$

where

$$
\hat{I}_{n}=\bar{\eta} I_{n}(\bar{\eta})-(-1)^{n} \bar{\eta} I_{n}(\bar{\eta})
$$

and

$$
\hat{J}_{n}=J_{n}(\bar{\eta})-(-1)^{n} J_{n}(\bar{\eta})
$$

The covariance function $K_{\bar{\zeta} \bar{\zeta}}(\tau)$ of $\bar{\zeta}$ is

$$
K_{\bar{\zeta} \bar{\zeta}}(\tau)=R_{\bar{\zeta} \bar{\zeta}}(\tau)-E^{2}[\bar{\zeta}]
$$

It may be verified that the term corresponding to $n=0$ in Eq. (29) of $R_{\bar{\zeta} \bar{\zeta}}(\tau)$ is in fact $E^{2}[\bar{\zeta}]$ so that

$$
K_{\bar{\zeta} \bar{\zeta}}(\tau)=\sigma^{2} \sum_{n=1}^{\infty} r^{n}\left(\hat{I}_{n}+\hat{J}_{n}\right)^{2}
$$

The terms in Eq. (33) converge rapidly so that only the first few terms should suffice. Retaining the first two terms in Eq. (33)

$$
\begin{aligned}
K_{\overline{\zeta \zeta}}(\tau)= & \sigma^{2}\left[r[1-Q(\bar{\eta})-Q(\overline{\bar{\eta}})]^{2}+\frac{r^{2}}{2}\right. \\
& {\left.[Z(\bar{\eta})-Z(\bar{\eta})]^{2}\right] }
\end{aligned}
$$

The approximate spectrum of $\bar{\zeta}$ is simply the Fourier transform of the approximate $K_{\zeta \bar{\zeta}}(\tau)$ and is

$$
\begin{aligned}
S_{\bar{\zeta} \bar{\zeta}}(\omega) \triangleq & S(\omega)[1-Q(\bar{\eta})-Q(\bar{\eta})]^{2}+\frac{S(\omega) * S(\omega)}{\sigma^{2}} \\
& \frac{[Z(\bar{\eta})-Z(\bar{\eta})]^{2}}{2}
\end{aligned}
$$

Where $\omega$ is frequency, $S(\omega)$ is frequency spectrum of $\zeta$ and is the fourier transform of $R(\tau)$ in Eq. (17). The symbol * denotes convolution.

Having found the spectrum $S_{\bar{\zeta} \bar{\zeta}}(\omega)$, we may compute 
the radiation stress component $S_{11}$ given by Battjes (1974)

$$
S_{11}=\rho g \int_{0}^{\infty}\left[\frac{1}{2}+\frac{2 k d}{\sinh 2 k d}\right] S_{\bar{\zeta} \bar{\zeta}}(\omega) d \omega
$$

where $\rho$ is density of water. From Eq. (13), the wave number of component wave in the finite water depth $k$ rederived as function of $k_{0}$, the component wave number in deep water. Then, applying the dispersion relationship $k_{0}=\frac{\omega^{2}}{g}$ the Eq. (36) becomes function of water depth $d$ and wave frequency $\omega$. The integration of Eq. (36) can be carried out numerically at given water depth.

\section{NUMERICAL RESULTS}

The expected values $E[\bar{\zeta}]$ of $\bar{\zeta}$ are plotted as a function of water depth $\mathrm{d}$ for $\omega_{0}=0.981,0.654,0.4905$ $\mathrm{Hz}$ and $\S=0.008$ in Fig. 2. Similar to the result of Yang (1991), the expected values $E[\bar{\zeta}]$ is non-positive in the whole range of water depth. This result can be explained by referring Eq. (3). It can be seen that in deep water breaking waves affected by the water depth is negligible, there the variation of $\bar{\zeta}$ from $\zeta$ is due to breaking wave in the range of $\zeta>\zeta_{b}$ so that the expected value of $\bar{\zeta}$ is small negative value called wave set-down. As wave propagate shoreward, magnitude of the expected value of $E[\bar{\zeta}]$ increase due to vigorous breaking. After breaking inception, $\mathrm{E}[\bar{\zeta}]$ vanish as water depth approaches to zero. The expected water surface elevation in the surf zone is called wave set-up.

As mention above, we choose the Wallops spectrum as the deep water spectrum. Therefore, the variance of ideal wave

$$
\sigma^{2}=\int S(\omega) d \omega=\int S_{o}(\omega) \frac{C_{g o}(\omega)}{C_{g}(\omega)} d \omega
$$

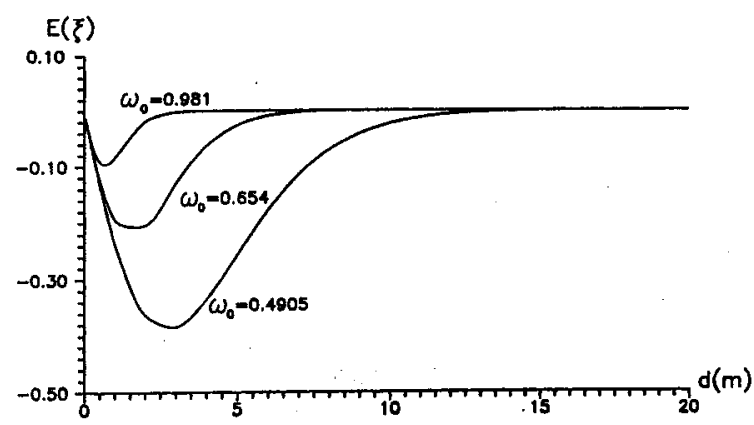

Fig. 2. Expected Values $E(\bar{\zeta})$ of $\bar{\zeta}$ as Function of Water Depth.
The variances $\sigma \frac{2}{y}$ and $\sigma^{2}$ are plotted as function of water 10832 and frequency of the waves at spectral peak $\omega_{0}=0.981,0.654$, and $0.4905 \mathrm{~Hz}$ in Fig. 3. The variances are increasing as water depth decreasing due to shoaling. The variance of ideal (fictitious) wave goes to infinite and $\sigma \frac{2}{\zeta}$ diminishes because of breaking. The breaking points are corresponding to the curves of slope equal to zero in Fig. 2 and 3.

The breaking spectra $S_{\bar{\zeta} \bar{\zeta}}(\omega)$ from Eq. (35) are plotted for $\xi=0.008$ and $\omega_{0}=0.981,0.654$, and 0.4505 $\mathrm{Hz}$ in Fig. 4, 5, and 6 respectively. These spectra are given at specified water depth $d=20,5,1$, and $0.5 \mathrm{~m}$. Since the wave energy dissipated at the surf zone, it is seen that the spectra vanishes at the water depth less than the depth of breaking points. Also, the main energy of breaking spectra move to low frequency as Hatori et al., (1981).

In Fig. 7 the radiation stress $S_{11}$ calculated from Eq. (36) are presented as functions of water depth for $\omega_{0}=0.981,0.654$, and 0.4509 respectively. The radiation stress obtained by Battjes (1974)

$$
S_{11}=\left[1-(2 \pi)^{1 / 2} Z(\sqrt{2} \bar{\eta})\right] S_{11 f}
$$

and

$$
\begin{gathered}
S_{11 f}=\rho_{g} \sigma^{2}\left(2 \overline{\bar{n}}-\frac{1}{2}\right) \\
\overline{\bar{n}}=\left[\int_{0}^{\infty}\left[\frac{1}{2}+\frac{k d}{\sinh (2 k d)}\right] S(\omega) d \omega\right] / \sigma^{2}
\end{gathered}
$$

also plotted in Fig. 7. The variations of radiation stress for Battjes (1974) and this study are due to the wide-band breaking model used in this study. By the mean time, the maghitude of these differences are increasing as energy spectra increasing. By the definition of the radiation stress

$$
\frac{d \bar{\zeta}}{d x}=-\frac{1}{\rho g(h+\bar{\zeta})} \frac{d S_{11}}{d x}
$$

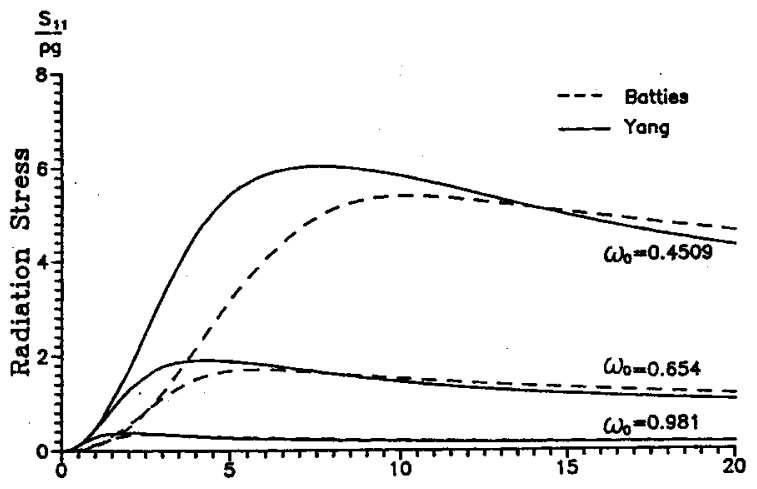
Fig. 3. Variances $\sigma \frac{2}{\zeta}$ and $\sigma^{2}$ of $\bar{\zeta}$ and $\zeta$ as Function of Water
Depth. 


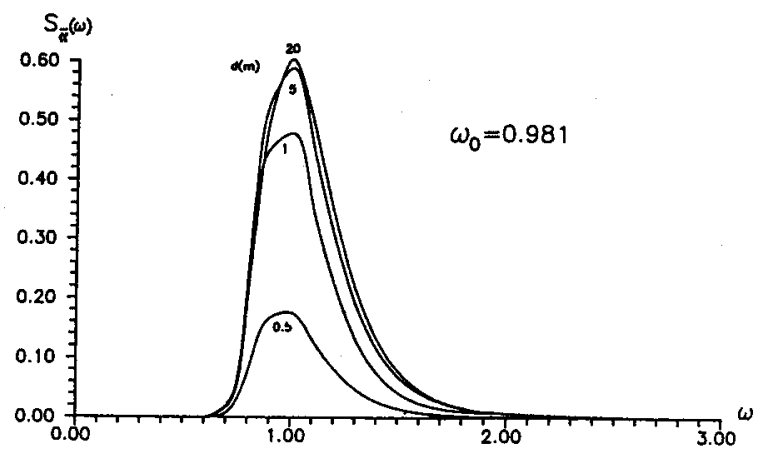

Fig. 4. Spectra $S_{\bar{\zeta}} \bar{\zeta}(\omega)$ Water Depth $d=20,5,1,0.5 \mathrm{~m}$ at $w_{0}=$ 0.981 .

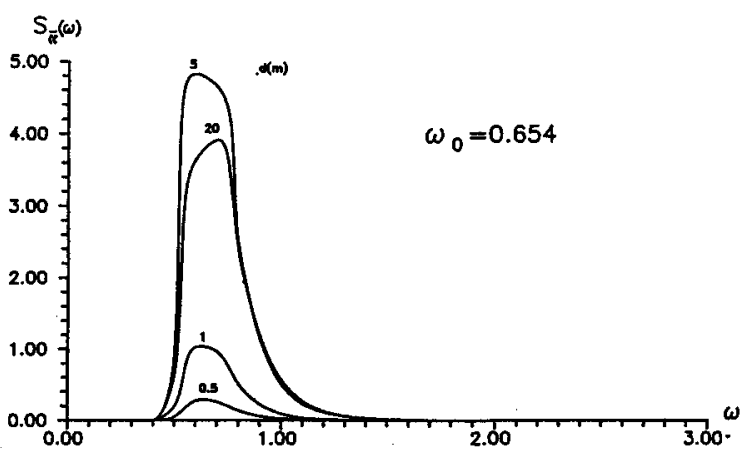

Fig. 5. Spectra $S_{\bar{\zeta}} \bar{\zeta}(\omega)$ Water Depth $d=20,5,1,0.5 \mathrm{~m}$ at $w_{0}=$ 0.654

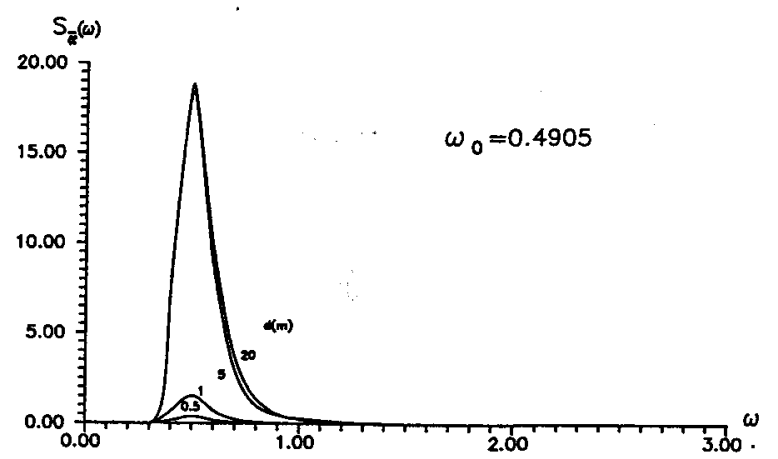

Fig. 6. Spectra $S \bar{\zeta} \bar{\zeta}(\omega)$ Water Depth $d=20,5,1,0.5 \mathrm{~m}$ at $w_{0}=$ 0.4905 .

it increase when the wave propagate from deep water to shallow water, the expected value of $\bar{\zeta}$ decrease, the water surface is set-down as Fig. 2. After breaking taking place, the wave energy is dissipated, therefore, radiation stress decrease. This is the wave set-up which is the mean water surface elevation in the surf zone.

\section{CONCLUSION AND DISCUSSION}

The breaking wave criterion for wave of finite band-width is establish (Eq. 1) by modifying the narrow-band breaking wave criterion used in Battjes

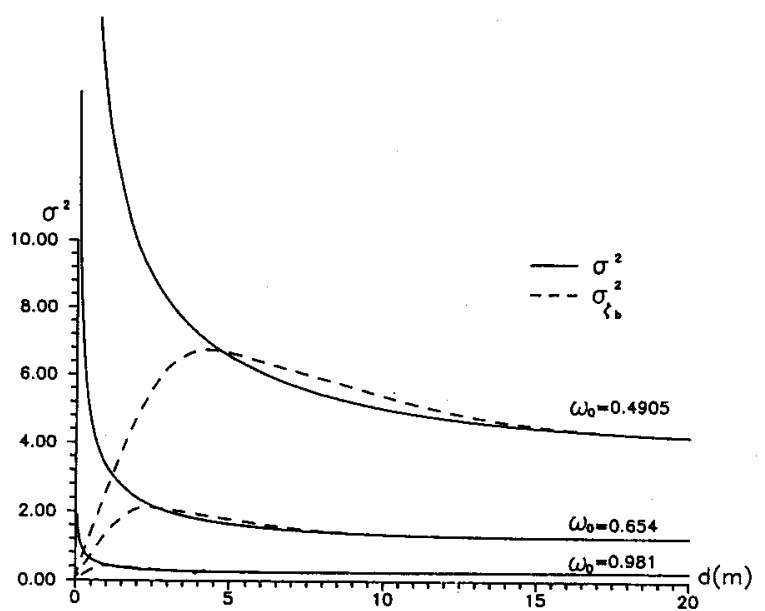

Fig 7. Radiation Stress $S_{11}$ as Function of Water Depth.

(1974) and the expression of component wave-number in deep water in terms of its in water of finite depth (Tayfun, 1979). Base on the breaking wave criterion, a model for breaking waves is proposed (Eq. 2). Assuming the ideal waves are stationary, zero-mean, and Gaussian (Pillips, 1977), the breaking wave spectrum is obtained (Eq. 35). It is noted that the spectrum $S_{\bar{\zeta} \bar{\zeta}}(\omega)$ of broken waves $\bar{\zeta}$ as given in Eq. (35) consists of two parts. Similar to the results of Yang (1991), the second part is negligible small compare with the first part so that $S_{\bar{\zeta} \bar{\zeta}}(\omega)$ is essentially given by

$$
S_{\bar{\zeta} \bar{\zeta}}(\omega)=S(\omega)[1-Q(\bar{\eta})-Q(\bar{\eta})]^{2}
$$

This mean that wave breaking reduces the spectrum $S(\omega)$ of the ideal wave $\zeta$ by the factor [ $1-\mathrm{Q}(\bar{\eta})$ $-\mathrm{Q}(\overline{\bar{\eta}})]^{2}$.

The model can be extended as the presence of current. The wave-current interaction in an opposing direction, the wave amplitude increase and wavelength decrease (Longuet-Higgins et al., 1961). As these circumstances, the sea surface becomes choppier and vigorous breaking takes place which increase the spectrum in high frequency (Huang et al. 1972). Wave breaking limits wave growth and reduces the spectral ordinates that evolves wave component into low frequency and has a similar spectral structure (Hatori, et al., 1981).

\section{REFERENCES}

1. Battjes, J. A. "Computation of set-up, longshore currents, run-up and overtopping due to windgenerated waves", Communications on Hydraulics, No. 74-2, Department of Civil Engineering, Delft University of Technology, Delft, The Netherlands, No. 74-2, 
1974.

2. Erdely A., W. Magnum, F. Oberhettinger, and F. G. Tricomi, "Higher Transcendental Functions", Vol. 2, McGraw-Hill Book Co. Inc., New York, N.Y., 1953.

3. Hatori, M., M. Tokuda, and Y. Toba, "Experimen tal study on strong interaction between regular wave and wind wave-I", J. Oceanogr. Soc. Japan, Vol. 37, pp. 111-119.

4. Huang, N. E., S. R. Long, C. C. Tung, and Y. Yuen, "A unified two-parameter wave spectral model for a general sea state", J. Fluid Mech. Vol. 112, 1981, pp. 203-224.

5. Huang, N. E., D. T. Chen, C. C. Tung, and J. Smith, "Interaction between Steady Non-Uniform Current and Gravity Waves with Applications for Current Measurements", Journal of Physical Oceanography, Vol. 2, No. 4, 1972, pp. 420-431.

6. Longuet-Higgins, M. S., and R. W. Steward, "The changes in Amplitude of Short Gravity Waves on Steady Non-Uniform Currents", Journal of Fluid Mechanics, Vol. 101, 1961, pp. 481-504.

7. Phillips, O. M., "The Dynamics of the Upper Ocean", Cambridge University Press, Cambridge, England, Second Edition, 1977, pp. 183-184.

8. Tayfun, M. A., "Distribution of Wave Heights Limited by Breaking", Civil Engineering in the Ocean, American Society of Civil Engineers, Vol. 1, 1979, pp336-343.

9. Tung, C. C. and N. E. Huang, "Spectrum of Breaking Waves in Deep Water", Journal of Engineering Mechanics, A.S.C.E., Vol. 113, No. 3., Mar. 1987, pp 293-302.

1O. Yang, W. H. "The breaking wave spectra in finite water depth", Proceedings of International Conference on Computer Modeling in Ocean 91 (eds. A.S. Arcilla et al.), A.A. Balkema, Rotterdam, Netherlands, pp. 181-187 (199 1) .

11. Yang, W. H. and C. C. Tung, "Statistical Properties of Random Wave in Surf Zone", Proceeding of The Third International Offshore and Polar Engineering Conference Singapore 6-11 June 1993.

\section{NOTATION}

$\begin{array}{ll}a_{b} & \begin{array}{l}\text { Amplitude of breaking wave } \\ \mathrm{C}_{\mathrm{go}}, C_{g}\end{array} \\ \begin{array}{l}\text { Group velocity in deep water and water } \\ \text { of finite depth respectively }\end{array} \\ \begin{array}{l}\text { Water depth } \\ E[\bullet]\end{array} & \begin{array}{l}\text { Expected value } \\ E_{1}, E_{2}\end{array} \\ \begin{array}{l}\text { Expected values computed in Eqs.(25) } \\ \text { and }(26)\end{array} \\ f_{\zeta}(\bullet) & \begin{array}{l}\text { Probability density function of zero-mean } \\ \text { Gaussian process }\end{array} \\ f_{\zeta_{1} \zeta_{2}(\bullet, \bullet)} & \text { Joint probability density function of zero- }\end{array}$

\begin{tabular}{|c|c|}
\hline$f_{\eta_{1} \eta_{2}}(\bullet \bullet)$ & $\begin{array}{l}\text { Joint probability density function of zero- } \\
\text { mean Gaussian processes } \eta_{1} \text { and } \eta_{2} \text { with } \\
\text { unit standard deviation }\end{array}$ \\
\hline$g$ & Gravitational acceleration \\
\hline$H(\bullet)$ & Heaviside step function \\
\hline$h_{\eta}(\bullet)$ & Hermite polynomial \\
\hline$K_{\overline{\zeta \zeta}}(\tau)$ & Covariance function of $\bar{\zeta}$ \\
\hline$k^{s b}$ & Wave-number \\
\hline$k_{\circ}$ & $\frac{W}{\omega_{0}^{2}}$ ave-number in deep water \\
\hline & $\begin{array}{l}\frac{10}{g} \\
\text { Integer index }\end{array}$ \\
\hline$\overline{\bar{n}}$ & $\begin{array}{l}\text { Integer index } \\
\text { Quantity defined in Eq.(43) }\end{array}$ \\
\hline$Q(\bullet)$ & $\begin{array}{l}\text { Quantity defined in Eq.(43) } \\
\text { Function defined in Eq.(12) }\end{array}$ \\
\hline$R(\bullet)$ & $\begin{array}{l}\text { Function defined in Eq.(12) } \\
\text { Autocorrelation function of } \zeta \text { (see } \\
\text { Eq.(18)) }\end{array}$ \\
\hline$R_{\bar{\zeta} \bar{\zeta}}(\tau)$ & Autocorrelation function of $\bar{\zeta}$ \\
\hline$r(\tau)$ & Correlation coefficient of $\zeta$ \\
\hline$S(\omega)$ & Spectrum of $\underline{\zeta}$ \\
\hline$S_{\bar{\zeta} \bar{\zeta}}(\omega)$ & Spectrum of $\bar{\zeta}$ \\
\hline$S_{0}^{\zeta}(\omega)$ & Spectrum of $\zeta$ in deep water (see Eq.(34)) \\
\hline$S_{11}$ & $\begin{array}{l}\text { Radiation stress component (see Eqs.(40) } \\
\text { and (41)) }\end{array}$ \\
\hline$S_{11 f}$ & $\begin{array}{l}\text { Radiation stress of fictitious wave } \zeta \text { (see } \\
\text { Eq. }(42) \text { ) }\end{array}$ \\
\hline$t$ & Time \\
\hline$U$ & Wind speed \\
\hline $\mathrm{Z}(\bullet)$ & $\begin{array}{l}\text { Standard zero-mean Gaussian probabil- } \\
\text { ity density function }\end{array}$ \\
\hline$\alpha$ & Nondimensional constant in Eq.(34) \\
\hline$\beta$ & Nondimensional constant in Eq.(34) \\
\hline & Elevation of ideal wave \\
\hline & Breaking limit of wave elevation \\
\hline$\zeta_{1}, \zeta_{2}$ & $\begin{array}{l}\text { Elevation of ideal wave at time instants } \\
t+\tau \text { and } t\end{array}$ \\
\hline & Elevation of breaking wave \\
\hline $\bar{\zeta}_{1}, \bar{\zeta}_{2}$ & $\begin{array}{l}\text { Elevation of breaking wave at time in- } \\
\text { stants } t+\tau \text { and } t\end{array}$ \\
\hline$\eta$ & Dummy variable \\
\hline$\eta_{1}, \eta_{2}$ & $\begin{array}{l}\text { Dummy variable; zero-mean Gaussian } \\
\text { processes with unit standard deviation at } \\
\text { time instants } t+\tau \text { and } t\end{array}$ \\
\hline
\end{tabular}

$\frac{\zeta_{b}}{\sigma}$

$\sigma$
$d$

$\frac{d}{\sigma}$

Dummy variable

Density of water

Standard deviation of $\zeta$

Standard deviation of $\bar{\zeta}$

Time lag

Radian frequency

$\frac{g}{U}$

Mean wave-frequency in deep water

$\bar{\omega}_{0}$

$I_{n}(\bullet), J_{n}(\bullet) \quad$ Quantities defined in Eqs.(21) and (22)

$I_{n}(\bullet), J_{n}(\bullet) \quad$ Quantities defined in Eqs.(28) and (29) 